\title{
Structural Criteria for the Rational Design of Selective Ligands. Convergent Hydrogen Bonding Sites for the Nitrate Anion
}

\author{
Benjamin P. Hay, * Maciej Gutowski \\ Chemical Sciences Division, Pacific Northwest National Laboratory, Richland, WA 99352 \\ David A. Dixon
}

Department of Chemistry, University of Alabama, Tuscaloosa, AL 35487

Jorge Garza and Rubicelia Vargas

Departamento de Química, División de Ciencias Básicas e Ingeniería, Universidad Autonóma Metropolitana-Iztapalapa. A.P. 55-534, México Distrito Federal 09340, México.

Bruce A. Moyer

Chemical Sciences Division, Oak Ridge National Laboratory, Oak Ridge, TN 37831

Supporting Information Available: Cartesian coordinates for the MP2/aug-cc-pVDZ optimized structures $1-\mathbf{6}$ and B3LYP/TZVP optimized structures $7-\mathbf{1 0}$, and potential energy surfaces for selected distortions of $\mathbf{5}$. 
Cartesian coordinates for the MP2/aug-cc-pVDZ optimized structures 1-6

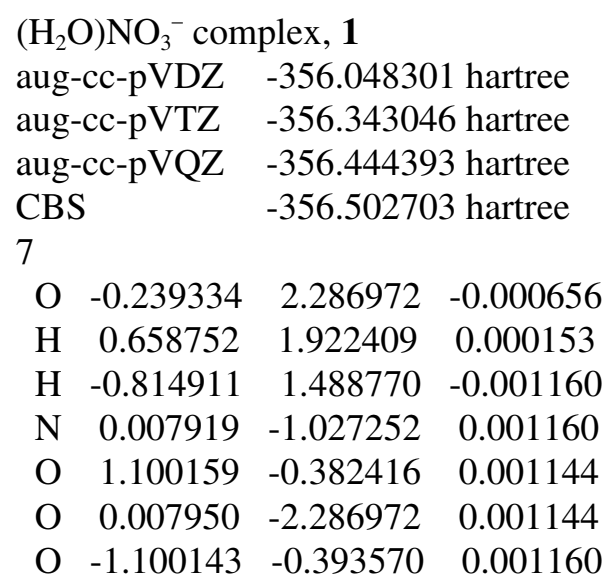

$\left(\mathrm{H}_{2} \mathrm{O}\right) \mathrm{NO}_{3}{ }^{-}$complex, 2

aug-cc-pVDZ -356.044082 hartree

aug-cc-pVTZ $\quad-356.338780$ hartree

aug-cc-pVQZ -356.440071 hartree

CBS $\quad-356.498344$ hartree

7

$\begin{array}{llll}\text { O } & 0.615112 & 2.334793 & -0.000839\end{array}$

$\mathrm{H} \quad 1.579361 \quad 2.305191 \quad 0.008316$

$\mathrm{H} \quad 0.351379 \quad 1.381134 \quad-0.004486$

$\begin{array}{llll}\mathrm{O} & 0.613983 & -0.443207 & -0.008316\end{array}$

$\mathrm{N} \quad-0.499008-1.073883 \quad-0.008179$

$\begin{array}{lllll}\mathrm{O} & -1.579346 & -0.420517 & -0.008331\end{array}$

O

$(\mathrm{MeOH}) \mathrm{NO}_{3}{ }^{-}$complex, 3

aug-cc-pVDZ -395.209502 hartree

aug-cc-pVTZ -395.542758 hartree

aug-cc-pVQZ -395.654989 hartree

CBS $\quad-395.719322$ hartree

10

C $\quad 0.598831 \quad 1.948578 \quad-0.005020$

$\begin{array}{llll}\mathrm{O} & -0.774170 & 1.585449 & -0.007172\end{array}$

$\begin{array}{llll}\mathrm{H} & -0.820633 & 0.597122 & -0.001389\end{array}$

$\mathrm{H} \quad 0.646774 \quad 3.049789 \quad-0.013321$

H $1.133972 \quad 1.562073 \quad-0.889236$

$\begin{array}{llll}\mathrm{H} & 1.127441 & 1.576126 & 0.889236\end{array}$

$\begin{array}{llll}\mathrm{N} & -0.028061 & -1.791718 & 0.016617\end{array}$

$\begin{array}{llll}\mathrm{O} & 1.058716 & -1.146057 & 0.002350\end{array}$

$\begin{array}{llll}\mathrm{O} & -0.036530 & -3.049805 & 0.030426\end{array}$

$\begin{array}{llll}\mathrm{O} & -1.133987 & -1.150894 & 0.017014\end{array}$

(MeOH) $\mathrm{NO}_{3}{ }^{-}$complex, 4

aug-cc-pVDZ -395.207473 hartree

aug-cc-pVTZ -395.540783 hartree

aug-cc-pVQZ -395.653037 hartree 


CBS
$\begin{array}{crrr}10 & & -395.717383 & \text { hartree } \\ \text { C } & 1.517136 & 1.803268 & -0.018677 \\ \text { O } & 0.099000 & 1.780640 & -0.031387 \\ \text { O } & 0.241318 & -0.966019 & -0.037476 \\ \text { N } & -0.870529 & -1.598770 & -0.037460 \\ \text { O } & -1.949463 & -0.943619 & -0.037476 \\ \text { O } & -0.870804 & -2.857590 & -0.037476 \\ \text { H } & -0.172836 & 0.831619 & -0.034760 \\ \text { H } & 1.834656 & 1.290115 & 0.907303 \\ \text { H } & 1.836258 & 2.857574 & -0.016052 \\ \text { H } & 1.949448 & 1.308319 & -0.907288\end{array}$

(NMF) $\mathrm{NO}_{3}{ }^{-}$complex, 5

aug-cc-pVDZ $\quad-488.451983$ hartree

aug-cc-pVTZ -488.861906 hartree

aug-cc-pVQZ -488.999354 hartree

CBS $\quad-489.078079$ hartree

13

$\begin{array}{lrrr}\mathrm{C} & 1.081635 & 2.112427 & 0.000000 \\ \mathrm{~N} & 0.020065 & 1.286743 & -0.000015 \\ \mathrm{C} & -1.335098 & 1.810760 & 0.000000 \\ \mathrm{H} & 2.046341 & 1.562378 & 0.000000 \\ \mathrm{H} & 0.179932 & 0.262451 & 0.000000 \\ \mathrm{H} & -2.046341 & 0.976257 & 0.000000 \\ \mathrm{O} & 1.032867 & 3.360413 & 0.000000 \\ \mathrm{H} & -1.511627 & 2.437653 & 0.889816 \\ \mathrm{H} & -1.511612 & 2.437637 & -0.889816 \\ \mathrm{O} & 0.714478 & -1.466431 & 0.000000 \\ \mathrm{O} & -1.478638 & -1.447159 & 0.000000 \\ \mathrm{~N} & -0.394455 & -2.104782 & 0.000015 \\ \mathrm{O} & -0.396866 & -3.360413 & 0.000015\end{array}$

$\left(\mathrm{CH}_{4}\right) \mathrm{NO}_{3}{ }^{-}$complex, 6

aug-cc-pVDZ -320.134942 hartree

aug-cc-pVTZ -320.407680 hartree

aug-cc-pVQZ -320.498880 hartree

CBS $\quad-320.551090$ hartree

9

$\begin{array}{llll}\text { O } & 1.087677 & -0.948929 & -0.007584\end{array}$

$\begin{array}{llll}\mathrm{N} & -0.011353 & -1.584488 & -0.007584\end{array}$

$\begin{array}{lllll}\mathrm{O} & -1.106186 & -0.944244 & -0.007584\end{array}$

$\begin{array}{llll}\text { O } & -0.013229 & -2.849594 & -0.007584\end{array}$

$\mathrm{H} \quad 0.184555 \quad 1.308868 \quad-0.010483$

$\begin{array}{llll}\text { C } & 0.100540 & 2.404877 & -0.007431\end{array}$

$\mathrm{H} \quad 1.106171 \quad 2.849594 \quad-0.019073$

$\mathrm{H} \quad-0.457840 \quad 2.736694 \quad-0.894424$

$\begin{array}{llll}\mathrm{H} & -0.435638 & 2.733154 & 0.894440\end{array}$ 
Cartesian coordinates for B3LYP/TZVP optimized structures 7-10

symmetric $(\mathrm{MeOH})_{3} \mathrm{NO}_{3}{ }^{-}$complex, 7, -627.822782 hartree

$\begin{array}{rrrr}22 & & & \\ \mathrm{~N} & 0.086853 & -1.133331 & -0.926331 \\ \mathrm{O} & 0.113739 & -2.396912 & -0.945053 \\ \mathrm{O} & -1.020096 & -0.524292 & -0.941162 \\ \mathrm{O} & 1.167450 & -0.479492 & -0.886948 \\ \mathrm{O} & -0.992706 & 2.309509 & -0.930389 \\ \mathrm{C} & 0.133743 & 2.747269 & -0.188522 \\ \mathrm{H} & -0.970657 & 1.329941 & -0.959534 \\ \mathrm{H} & 0.214615 & 3.830902 & -0.315704 \\ \mathrm{H} & 1.060883 & 2.278473 & -0.533813 \\ \mathrm{H} & 0.031143 & 2.537766 & 0.886246 \\ \mathrm{O} & 3.609665 & -1.917419 & -0.841843 \\ \mathrm{C} & 3.399887 & -3.141953 & -0.158081 \\ \mathrm{H} & 2.749908 & -1.448898 & -0.888931 \\ \mathrm{H} & 4.307968 & -3.742508 & -0.266235 \\ \mathrm{H} & 2.552521 & -3.701645 & -0.567261 \\ \mathrm{H} & 3.216782 & -2.992752 & 0.915985 \\ \mathrm{O} & -2.355560 & -3.795792 & -1.024948 \\ \mathrm{C} & -3.336472 & -3.018417 & -0.358536 \\ \mathrm{H} & -1.515976 & -3.289474 & -1.021240 \\ \mathrm{H} & -4.307983 & -3.491760 & -0.529510 \\ \mathrm{H} & -3.371109 & -1.990234 & -0.732941 \\ \mathrm{H} & -3.166016 & -2.974594 & 0.727081\end{array}$

asymmetric $(\mathrm{MeOH})_{3} \mathrm{NO}_{3}{ }^{-}$complex, 8, -627.822272 hartree 22

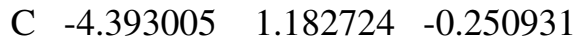

$\begin{array}{llll}\text { O } & -3.533279 & 1.916397 & -1.106110\end{array}$

$\mathrm{H} \quad-5.404800 \quad 1.579132-0.378143$

$\begin{array}{llll}\mathrm{H} & -4.117325 & 1.289902 & 0.808441\end{array}$

$\begin{array}{llll}\mathrm{H} & -4.396881 & 0.114258 & -0.490875\end{array}$

$\mathrm{H} \quad-2.659119 \quad 1.473450 \quad-1.103546$

$\begin{array}{llll}\mathrm{N} & -0.955414 & -0.588409 & -0.921936\end{array}$

$\begin{array}{llll}\text { O } & -2.029968 & -1.225174 & -0.918350\end{array}$

$\begin{array}{llll}\mathrm{O} & -0.968475 & 0.689240 & -0.981415\end{array}$

O $\quad 0.159592 \quad-1.181824-0.862686$

$\begin{array}{llll}\mathrm{O} & 1.560608 & 2.002518 & -0.969345\end{array}$

C $\quad 2.424545 \quad 1.277252 \quad-0.110489$

$\mathrm{H} \quad 0.706116 \quad 1.525085 \quad-1.008469$

$\begin{array}{llll}\mathrm{H} & 3.425400 & 1.708862 & -0.205368\end{array}$

$\begin{array}{llll}\mathrm{H} & 2.468597 & 0.215408 & -0.373398\end{array}$

$\begin{array}{llll}\mathrm{H} & 2.121307 & 1.351700 & 0.944168\end{array}$

O $\quad 0.134811-4.006241-0.715683$

C $\quad-0.995697 \quad-4.390503 \quad 0.048203$

$\mathrm{H} \quad 0.118332 \quad-3.029602 \quad-0.804474$

$\mathrm{H} \quad-1.075790 \quad-5.480560 \quad-0.001465$

$\mathrm{H} \quad-1.920944 \quad-3.948303 \quad-0.335968$

H $\quad-0.900100 \quad-4.105042 \quad 1.106125$ 
symmetric $(\mathrm{NMF})_{3} \mathrm{NO}_{3}{ }^{-}$complex, 9, -908.366879 hartree 31

$\begin{array}{rrrr}\mathrm{C} & 3.459625 & -4.312698 & 0.104813 \\ \mathrm{~N} & 3.319412 & -2.976654 & 0.092209 \\ \mathrm{C} & 4.461761 & -2.078186 & 0.123184 \\ \mathrm{H} & 2.486740 & -4.833908 & 0.076614 \\ \mathrm{H} & 2.379639 & -2.576462 & 0.059616 \\ \mathrm{H} & 4.110092 & -1.048569 & 0.105682 \\ \mathrm{O} & 4.527924 & -4.925278 & 0.142502 \\ \mathrm{H} & 5.057175 & -2.232208 & 1.027008 \\ \mathrm{H} & 5.114807 & -2.244751 & -0.737595 \\ \mathrm{O} & 0.553574 & -1.940735 & -0.002106 \\ \mathrm{O} & 1.632965 & -0.037003 & 0.025223 \\ \mathrm{~N} & 0.544083 & -0.677094 & -0.007600 \\ \mathrm{O} & -0.554077 & -0.053436 & -0.049561 \\ \mathrm{C} & -4.066681 & -1.377441 & -0.139618 \\ \mathrm{~N} & -2.838943 & -1.922638 & -0.119400 \\ \mathrm{C} & -2.630508 & -3.361252 & -0.130554 \\ \mathrm{O} & -5.130814 & -1.997559 & -0.169189 \\ \mathrm{H} & -4.032532 & -0.273926 & -0.127090 \\ \mathrm{H} & -2.023315 & -1.307068 & -0.095154 \\ \mathrm{H} & -1.562805 & -3.570129 & -0.110077 \\ \mathrm{H} & -3.099091 & -3.831848 & 0.737915 \\ \mathrm{H} & -3.063568 & -3.812958 & -1.027008 \\ \mathrm{C} & 2.235992 & 3.662018 & -0.018707 \\ \mathrm{~N} & 1.145752 & 2.877090 & -0.006943 \\ \mathrm{C} & -0.201340 & 3.423309 & -0.004929 \\ \mathrm{O} & 2.237396 & 4.893921 & -0.029100 \\ \mathrm{H} & 3.171387 & 3.075516 & -0.017502 \\ \mathrm{H} & 1.265106 & 1.862137 & 0.001907 \\ \mathrm{H} & -0.920517 & 2.606735 & 0.005280 \\ \mathrm{H} & -0.366592 & 4.055649 & 0.871536 \\ \mathrm{H} & -0.377869 & 4.034836 & -0.893800\end{array}$

asymmetric $(\mathrm{NMF})_{3} \mathrm{NO}_{3}{ }^{-}$complex, 10, -908.366629 hartree 31

$\begin{array}{llll}\text { C } & 2.913086 & 3.288956 & -0.006104\end{array}$

N $\quad 1.678986 \quad 2.757034 \quad-0.008300$

C $\quad 0.482254 \quad 3.582123 \quad-0.008911$

$\begin{array}{llll}\text { O } & 3.178848 & 4.491714 & -0.004379\end{array}$

$\begin{array}{llll}\mathrm{H} & 3.700867 & 2.515518 & -0.005936\end{array}$

$\mathrm{H} \quad 1.577271 \quad 1.740646-0.009674$

$\begin{array}{llll}\mathrm{H} & -0.380112 & 2.918747 & -0.010193\end{array}$

$\begin{array}{llll}\mathrm{H} & 0.449997 & 4.225723 & 0.874207\end{array}$

$\mathrm{H} \quad 0.451660 \quad 4.226883 \quad-0.891220$

O $\quad 1.520600 \quad-0.206741-0.012222$

$\begin{array}{llll}\mathrm{O} & -0.611450 & 0.285568 & -0.013779\end{array}$

$\mathrm{N} \quad 0.303741 \quad-0.586258-0.012253$

O $\quad 0.028336-1.808228-0.010803$

C $\quad-4.330765-0.211761-0.008102$ 


$\begin{array}{cccc}\mathrm{N} & -3.260468 & -1.023956 & -0.010605 \\ \mathrm{C} & -3.389038 & -2.471756 & -0.011200 \\ \mathrm{O} & -5.509033 & -0.571732 & -0.006073 \\ \mathrm{H} & -4.044571 & 0.854492 & -0.008072 \\ \mathrm{H} & -2.325165 & -0.611588 & -0.012024 \\ \mathrm{H} & -2.386871 & -2.895630 & -0.015274 \\ \mathrm{H} & -3.930862 & -2.817184 & 0.873337 \\ \mathrm{H} & -3.937729 & -2.815659 & -0.892090 \\ \mathrm{C} & 4.628845 & -2.352707 & -0.016449 \\ \mathrm{~N} & 3.304825 & -2.582718 & -0.006836 \\ \mathrm{C} & 2.755249 & -3.928406 & 0.006195 \\ \mathrm{O} & 5.509018 & -3.214386 & -0.015320 \\ \mathrm{H} & 4.865463 & -1.274384 & -0.025879 \\ \mathrm{H} & 2.662521 & -1.788422 & -0.008972 \\ \mathrm{H} & 1.670441 & -3.845139 & 0.009506 \\ \mathrm{H} & 3.078812 & -4.491700 & -0.873138 \\ \mathrm{H} & 3.085434 & -4.477493 & 0.892090\end{array}$


Potential energy surfaces (B3LYP/TZVP+) for selected distortions of 5.
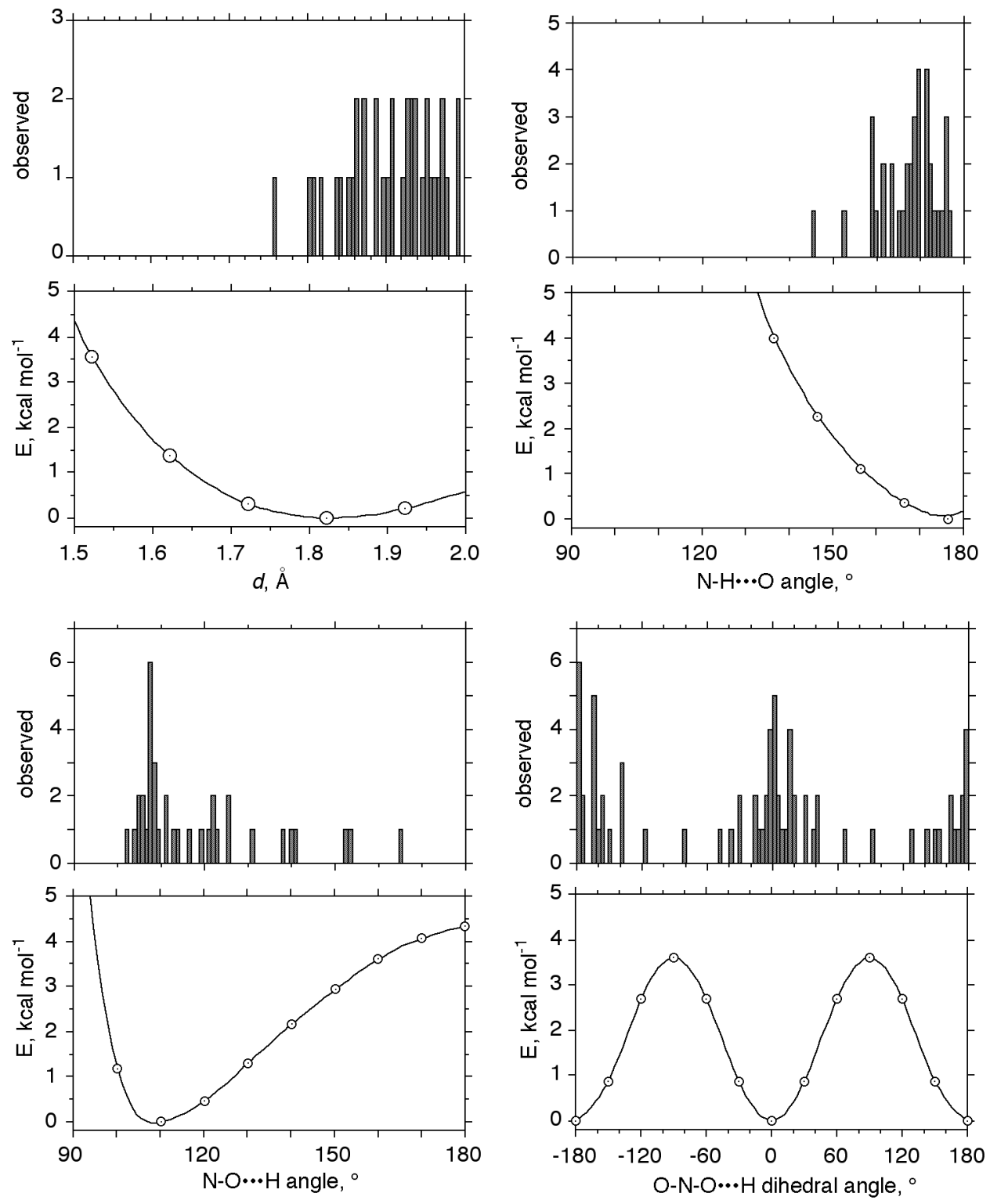

A comparison of experimental distributions of geometric parameters for all amide $\mathrm{NO}_{3}{ }^{-}$hydrogen bonds with potential energy surfaces obtained at the B3LYP/TZVP+ level of theory for the $\left(\mathrm{NMF} \mathrm{NO}_{3}{ }^{-}\right.$ complex, 5: $d$ (top left), $\mathrm{N}-\mathrm{H} \bullet \bullet \cdot \mathrm{O}$ angle (top right), $\mathrm{N}-\mathrm{O} \bullet \bullet \cdot \mathrm{H}$ angle (bottom left), $\mathrm{O}-\mathrm{N}-\mathrm{O} \bullet \bullet \cdot \mathrm{H}$ dihedral angle (bottom right). 